\title{
Sept grands marchands catholiques français participant au commerce avec la Nouvelle-France (1660-1715)
}

\section{J. F. Bosher}

Volume 48, numéro 1, été 1994

URI : https://id.erudit.org/iderudit/305298ar

DOI : https://doi.org/10.7202/305298ar

Aller au sommaire du numéro

Éditeur(s)

Institut d'histoire de l'Amérique française

ISSN

0035-2357 (imprimé)

1492-1383 (numérique)

Découvrir la revue

Citer cet article

Bosher, J. F. (1994). Sept grands marchands catholiques français participant au commerce avec la Nouvelle-France (1660-1715). Revue d'histoire de l'Amérique française, 48(1), 3-27. https://doi.org/10.7202/305298ar
Résumé de l'article

Pendant le règne de Louis XIV, sept grands marchands français de religion catholique s'imposèrent dans le commerce avec la Nouvelle-France à un moment où les marchands huguenots armaient de moins en moins de navires. On a souvent exagéré le rôle de ces derniers marchands pendant cette période, et il est probable que leur importance ait diminué à la suite des persécutions dont ils ont été victimes pendant les guerres de 1689 à 1713. En effet, la monarchie se montrait très méfiante à leur égard, une situation dont les marchands catholiques ont pu bénéficier. D'origine modeste, les sept marchands catholiques qui sont l'objet de notre étude ont été favorisés par cinq facteurs : des mariages avantageux, des relations solides de famille ou d'affaires outre-Atlantique, des rapports étroits avec des membres du clergé ou des officiers métropolitains (dans un cas au moins) et des occasions d'affaires issues des guerres de Louis XIV. Malheureusement, ces marchands subirent de lourdes pertes financières, assez fréquentes en ces années, car les Bourbons ne payaient pas toujours leurs dettes.
Tous droits réservés @ Institut d'histoire de l'Amérique française, 1994
Ce document est protégé par la loi sur le droit d'auteur. L'utilisation des services d'Érudit (y compris la reproduction) est assujettie à sa politique d'utilisation que vous pouvez consulter en ligne.

https://apropos.erudit.org/fr/usagers/politique-dutilisation/ 


\title{
SEPT GRANDS MARCHANDS CATHOLIQUES FRANÇAIS PARTICIPANT AU COMMERCE AVEC LA NOUVELLE-FRANCE (1660-1715)
}

\author{
J. F. BOSHER \\ Département d'histoire \\ Université York
}

\section{RÉSUMÉ}

Pendant le règne de Louis XIV, sept grands marchands français de religion catholique s'imposèrent dans le commerce avec la Nouvelle-France à un moment où les marchands huguenots armaient de moins en moins de navires. On a souvent exagéré le rôle de ces derniers marchands pendant cette période, et il est probable que leur importance ait diminué à la suite des persécutions dont ils ont été victimes pendant les guerres de 1689 à 1713 . En effet, la monarchie se montrait très méfiante à leur égard, une situation dont les marchands catholiques ont pu bénéficier. D'origine modeste, les sept marchands catholiques qui sont l'objet de notre étude ont été favorisés par cinq facteurs: des mariages avantageux, des relations solides de famille ou d'affaires outre-Atlantique, des rapports étroits avec des membres du clergé ou des officiers métropolitains (dans un cas au moins) et des occasions d'affaires issues des guerres de Louis XIV. Malheureusement, ces marchands subirent de lourdes pertes financières, assez fréquentes en ces années, car les Bourbons ne payait pas toujours leurs dettes.

\begin{abstract}
Seven great Catholic merchants trading with New France in the reign of Louis $X I V$ sent many ships across the Atlantic, and their importance in this trade suggests that the Huguenot merchants were sending fewer and fewer. The role of big Huguenot shipping merchants in this period is sometimes exaggerated; it was probably reduced as a result of persecution. Men of humble origin, the seven merchants in question had the benefit of five factors: advantageous marriages, strong business or personal relationships in Canada usually resulting from visits there, close relations in the Church, or with colonial officials (in one case, at least), and wartime opportunities. The religious conflict in the wars of 1689-1713 made the French government wary of Huguenot merchants and so improved the chances of Catholic merchants, even humble ones. Unfortunately for them, however, they suffered losses usual in the Bourbon monarchy, losses due to the government's failure to pay its debts.
\end{abstract}


Étudier le milieu des gens d'affaires qui s'occupaient du commerce avec le Canada sous le règne de Louis XIV n'est pas une mince tâche. Si la liste des navires susceptibles d'être identifiés dans les diverses sources disponibles au Canada et en France ne peut jamais prétendre être complète, retracer leurs armateurs et leurs propriétaires s'avère également un travail extrêmement fastidieux. Nous avons traité ailleurs des sources, problèmes et écueils qui attendent les chercheurs dans ce domaine de l'histoire du Canada ${ }^{1}$. Cependant, il nous apparaît intéressant de dégager les traits caractéristiques de certains de ces gens d'affaires même si on ne peut en dresser la liste complète. Notre plus récente compilation de navires nous permet de repérer les noms de 443 vaisseaux qui traversèrent l'Atlantique de 1660 à 1715, en plus des noms de quelque 120 personnes qui les armaient ou qui en étaient les propriétaires. Les noms d'à peu près la moitié de ces armateurs et propriétaires, soit une soixantaine, paraissent plus d'une fois dans les documents relatifs à ce commerce, mais les origines et le milieu social de plusieurs de ces personnes échappent toujours à notre recensement. Malgré les lacunes, un groupe de sept marchands nous semble digne de l'attention des historiens à cause de leur succès, leurs origines modestes et leur foi catholique. Même si ce ne sont pas les seuls marchands à rencontrer ces critères, ils méritent qu'on s'intéresse à eux vu le rôle très important qu'ils jouèrent dans le commerce sous le règne de Louis XIV. Ils armaient ou possédaient pas moins de 123 navires engagés dans le commerce avec la NouvelleFrance (presque tous destinés à Québec), ce qui représente $27,7 \%$ des vaisseaux que nous avons identifiés ${ }^{2}$.

Les sept négociants français que nous avons retenus sont remarquables car, même issus de milieu très modeste, parfois pauvre, ils sont parvenus à s'imposer dans le grand commerce avec le Canada. Le plus ancien, Antoine Grignon (ca 1612-1775), était un petit marchand illettré de La Rochelle qui ne signa que par «AG» toute sa vie, mais dont les créances au Canada totalisaient à son décès 11246 livres $^{3}$. Pour sa part, Guillaume Jung (1637-1710), né le 27 juin 1637 dans une famille de petits marchands de Fleurance (Gers), une petite ville assez éloignée de la mer, a fondé une dynastie de négociants qui firent

1. J. F. Bosher, Négociants et navires du commerce avec le Canada de 1660 à 1760: dictionnaire biographique (Ottawa, Ministère de l'Environnement, 1992), introduction.

2. Nous avons repéré ces navires à partir des sources suivantes: Archives départementales de la Charente-Maritime [AD], Archives départementales de la Gironde, Archives nationales du Québec, Archives nationales de la France, multiples documents détaillés dans J. F. Bosher, op. cit. (Voir graphique).

3. AD Charente-Maritime à La Rochelle, minutes de 17 pages du notaire Teuleron, 18 décembre 1675 , intitulé «inventaire après décès». 
Nombre de navires affrétés par les sept marchands et nombre total de navires destinés à la Nouvelle-France

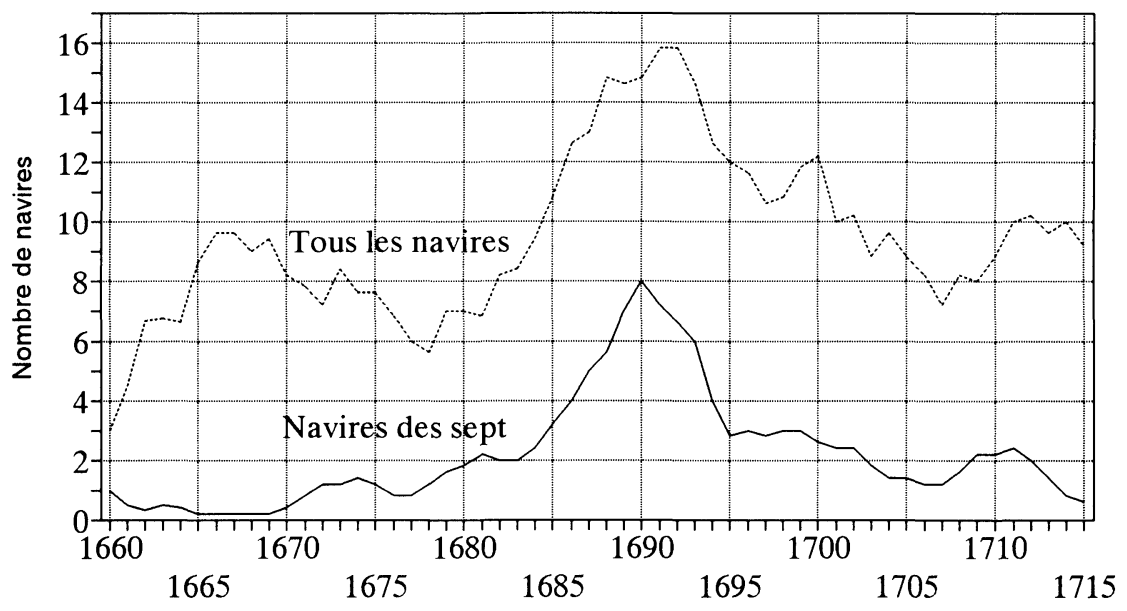

Moyenne mobile de cinq ans

le commerce transatlantique jusqu'en $1753^{4}$. Le troisième de nos sept négociants, Hilaire Bourgine (1650-1725), était également le fils d'un petit marchand d'une ville de l'intérieur, Poitiers en l'occurrence, où il fut baptisé le 5 janvier 1650. En 1687, il épousa la fille d'un vitrier qui ne lui apporta que 1000 livres de $\operatorname{dot}^{5}$. Mais son succès fut tel dans son commerce avec le Canada qu'en 1722, il put offrir à son fils, Charles-Polycarpe Bourgine (1696-1756), déjà établi à La Rochelle comme lui, une dot de 10000 livres $^{6}$. Le quatrième négociant, Jean Gitton (1633-1690), naquit à La Rochelle; son père était un boulanger de la paroisse Saint-Nicolas, située près du port, et il vendait du «pain bis-cuit» aux capitaines de navires. Dans les années 1680, avec son père, il était devenu propriétaire de plusieurs navires, d'une maison à Montréal et de plusieurs propriétés à La Rochelle ${ }^{7}$.

4. Voir notre article, «Une famille de Fleurance dans le commerce du Canada à Bordeaux (1683-1753): les Jung», Annales du Midi, 95,162 (1983): 159-184.

5. AD Charente-Maritime, notaire Berthellot (La Rochelle), registres, 9 juin 1687, mariage.

6. Bibliothèque municipale de Poitiers, registres de la paroisse Saint-Jean-Baptiste (Poitiers), registre 161, folio 58, verso, 5 janvier 1650, baptême; Archives départementales de la Vienne (Poitiers), E 427 202, notaire Guillaume de Cressac (Poitiers), 9 novembre 1722, contrat de mariage. Voir pour des notes et références plus complètes sur ces marchands notre ouvrage, Négociants et navires..., op. cit. 
Les trois autres marchands débutèrent comme simple engagé lorsqu'ils émigrèrent au Canada. Antoine Pascaud (1665-1717), sans doute le mieux connu, est né dans le village de La Prade, tout près d'Aubeterre-sur-Drônne (Charente), où son père était tanneur ${ }^{8}$. Il est passé au Canada avant le mois d'octobre 1684 comme engagé, paraîtil. Son succès dans le commerce avec la colonie, assez bien connu d'ailleurs, a été éblouissant ${ }^{9}$. Les sixième et septième de nos négociants, à peu près inconnus des historiens, étaient probablement, au début, les protégés de Guillaume Jung. L'un d'eux, Paul Berry (16651709), est né le 15 mai 1665 à Fleurance, la ville natale de Jung, et, le 5 avril 1685, il signa un contrat d'engagement avec Joseph Lombard, commissaire de la marine, pour travailler au Canada ${ }^{10}$. Pierre Plassan signa lui aussi, le 14 avril 1692 à Bordeaux, un contrat d'engagé avec ce même Guillaume Jung; il devait passer deux ans à Québec, à 75 livres par an, sous les ordres du fils et du neveu de Jung, tous deux nommés Jean Jung ${ }^{11}$. Pierre Plassan, né le 15 août 1670 était le fils d'un charpentier de barriques, Micheau Plassan, et de Peyronne Faure, du village de Baurech (Entre-deux-mers) près de Bordeaux ${ }^{12}$. Quoique simples engagés à l'origine, Plassan et Berry sont rapidement devenus des négociants expédiant navires et cargaisons de marchandises au Canada et en France.

Ces sept marchands n'étaient pas tous contemporains, leurs dates de naissance s'échelonnant de 1612 (ca.) à 1670; ils ont été actifs pendant les années où le commerce a progressé au gré des projets impériaux de Louis XIV. Entre 1660 et 1715 donc, l'activité commerciale de ces catholiques talentueux et évidemment ambitieux prend de plus en plus d'ampleur. Cependant, les sources ne permettent pas de quantifier ce commerce en tous ses détails, mais elles nous

7. Jean Gitton a passé son enfance dans une famille de boulangers (AD CharenteMaritime, notaire Morry (La Rochelle), 26 janvier 1658, inventaire de sa mère, Marie Lauvanson, veuve de Jean Gitton, père; notaire Teuleron (La Rochelle), registres, 22 avril 1635, folio 56-57, mariage d'une sœur avec un boulanger (Isaac Basset); notaire Cherbonnier (La Rochelle), registres, 9 septembre 1648, folio 128, vente de biscuit; notaire Berthellot (La Rochelle), 22 août 1690, inventaire après le décès de Jean Gitton).

8. $\mathrm{AD}$ Charente (à Angoulême), registres de la paroisse Notre-Dame de La Prade, $1^{\text {er }}$ février 1665 , baptême.

9. Pascaud a signé un document (en tant que témoin) à Québec le 31 octobre 1684 (Archives nationales du Québec, notaire Rageot (Québec), 31 octobre 1684, Procès-verbal de délibération de la Compagnie de la Baie d'Hudson...).

10. Fleurance (Gers), archives municipales, G.G. 5 B, 16 mai 1665, baptême; AD Gironde, notaire Belso-Dubos (Bordeaux), 5 avril 1685 [3 E 15286], engagement.

11. AD Gironde, notaire Loste (Bordeaux), 14 avril 1692, folio 59, engagement.

12. Cyprien Tanguay se trompe sur les origines de Plassan. Voir à la mairie de Baurech, GG4, folios 25 et 30; AD Gironde, notaire Loste (Bordeaux), 14 avril 1692, engagement. 
permettent toutefois d'établir un tableau général de leurs activités (voir annexe) ${ }^{13}$.

Malgré l'importance de leur carrière respective, c'est à peine si l'on connaît les noms de ces négociants et quelques détails relatifs à leur commerce. D'ailleurs, une partie des spécialistes du commerce en Nouvelle-France ont tendance à minimiser le rôle de ces catholiques en faisant grand cas des vieilles familles protestantes ${ }^{14}$. Certains historiens ont même tendance à exagérer le rôle de ces protestants. Par exemple, le négociant catholique Jean Gitton (1633-1690) fut présenté par le distingué historien Pierre Boissonnade comme membre de la famille huguenote Guitton, connue à cause de Jean Guitton, le maire de La Rochelle qui dirigeait la défense de la ville pendant le siège de $1627-1628^{15}$. Or, cette identification erronée - une des rares dans les travaux de cet excellent chercheur - a eu d'autant plus de conséquences que les études portant sur les marchands de La Rochelle au dix-septième siècle s'avèrent extrêmement rares. Un deuxième exemple de confusion se trouve dans une autre étude, sur les marchands de Bordeaux cette fois, soit la thèse souvent et justement citée d'un chercheur américain, Jonathan Howes Webster. Selon Webster, l'un des grands marchands catholiques dans le commerce bordelais avec le Canada à partir des années 1670, Guillaume Jung (1637-1710), était «a naturalized Dutch merchant who was probably of Protestant background ${ }^{16} \gg$. Mais nos recherches ont prouvé qu'au contraire Jung et ses ancêtres, étaient des catholiques de la ville de Fleurance ${ }^{17}$. Le seul transfert de ces deux commerçants, Gitton et Jung, du côté des catholiques - «anciens catholiques» comme on disait à l'époque bouleverse le bilan religieux des expéditions, car ils étaient très actifs dans le commerce transatlantique.

De telles confusions proviennent sans doute des plaintes qu'on trouve un peu partout dans les documents d'époque. Ainsi, peut-on

13. Archives départementales de la Charente-Maritime, Archives départementales de la Gironde, Archives nationales du Québec, Archives nationales de la France, multiples documents détaillés dans J. F. Bosher, Négociants et navires du commerce avec le Canada de 1660 à 1760 (Ottawa, Ministère de l'Environnement, 1992).

14. Marcel Delafosse, «La Rochelle et le Canada au XVII ${ }^{\mathrm{e}}$ siècle», Revue d'histoire de l'Amérique française, 4,3 (décembre 1951): 476, 479, 483-84; Marcel Delafosse, Histoire de La Rochelle (Toulouse, Privat, 1985), 164.

15. Pierre Boissonnade, «La marine marchande, le port et les armateurs de La Rochelle à l'époque de Colbert (1662-1683)», Bulletin du Comité des travaux historiques et scientifiques, section de géographie, 37 (1922): 2, 14-15, 21, 26-30; Marcel Delafosse, Histoire de La Rochelle, 147-148, 152-153, 156.

16. J. H. Webster, «The Merchants of Bordeaux in Trade to the French West Indies, 1664-1717», thèse de doctorat inédite de l'Université du Minnesota, 1972, 243.

17. J. F. Bosher, «Une famille de Fleurance», op. cit., 163. 


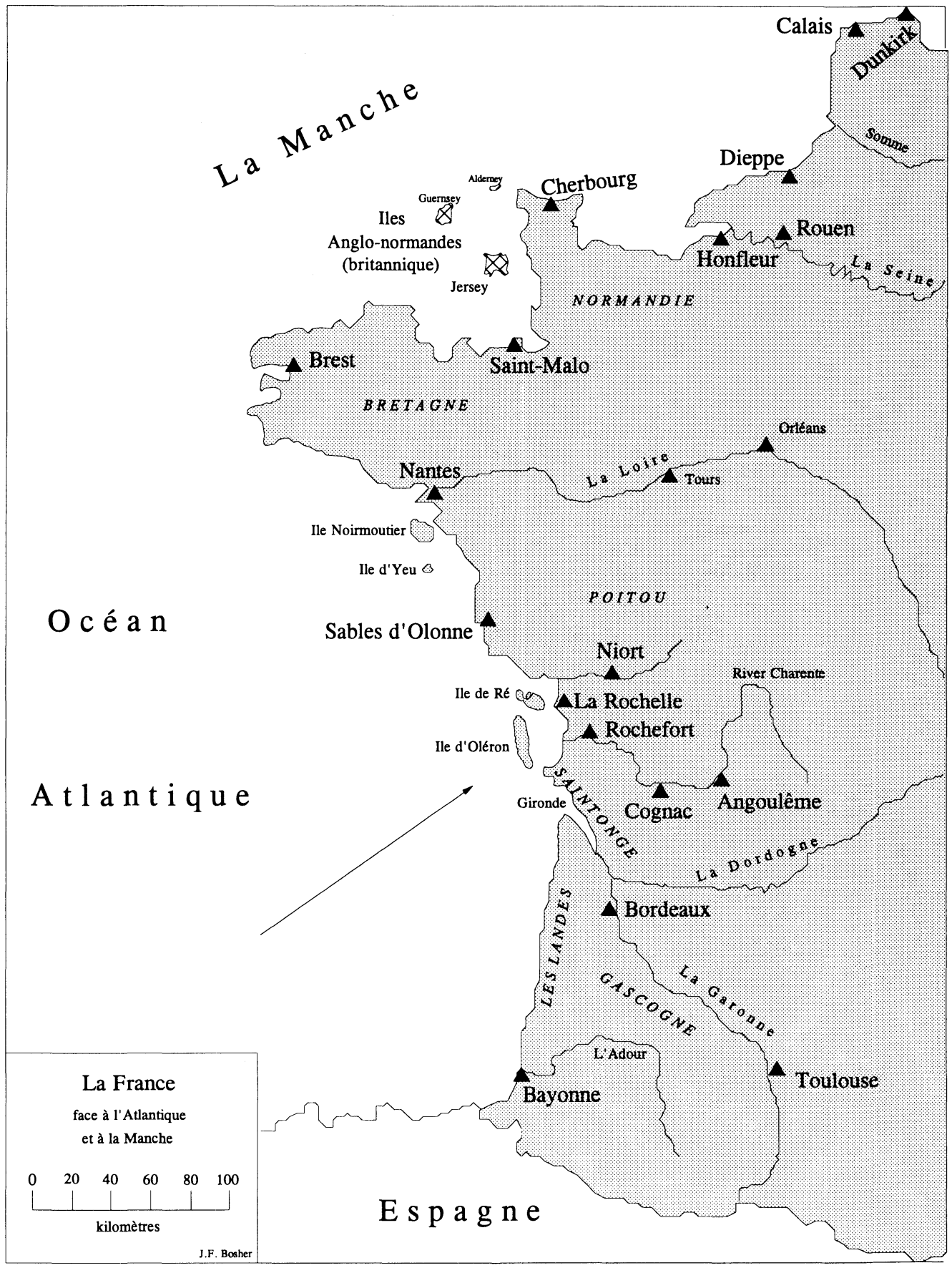


lire dans un Mémoire de l'évesque de Québec sur les Protestans (1670) «que les commerçants de France envoyent des commis Protestans, que depuis longtemps le clergé en a fait connoistre les inconvénients et par rapport à la religion et par rapport à l'état ${ }^{18}{ }^{\text {» }}$. Dans le même esprit, Jean Dudouyt, grand vicaire de l'évêque de Québec, écrit au ministère en 1681 contre Clerbaut Bergier de la Compagnie de la pêche sédentaire en Acadie parce que ces derniers «sont tous Huguenots et mènent un ministre ${ }^{19}{ } \gg$. Deux ans plus tard, à Québec, l'intendant De La Barre se plaint lui aussi d'un «nommé Bergier, de la Rochelle, Huguenot fort opiniastre ${ }^{20} \gg$. De même, le 10 novembre 1683, l'évêque écrit de nouveau à Versailles pour se plaindre «qu'il est passé en ce lieu-là [Port-Royal, Acadie] des hérétiques de la Rochelle qui prétendent s'y établir ... et qui ont desjà trop de communication avec les Anglois de Baston ${ }^{21} \gg$. En effet, plusieurs des marchands réfugiés à Boston, New York et ailleurs dans les colonies anglaises étaient connus comme étant d'origine rochelaise ${ }^{22}$. À ces récriminations s'ajoutent les doléances de marchands catholiques contre les marchands huguenots. Le 31 mai 1691, plusieurs négociants impliqués dans le commerce avec le Canada portaient plainte contre Jean Massiot, ancien huguenot converti. On peut lire par exemple:

Le nommé Grignon se plaint que ledit sieur Massiot sous prétexte du service de Sa Majesté abuse du pouvoir qu'il a en chargeant souvent pour son compte sous le nom de sa femme et de son fils comme ledit Grignon dit luy estre arrivé l'année dernière. Le sieur Massiot avait un méchant navire nommé Le Maréchal qu'il chargea pour le Roy de farines et de lard pour les Isles de l'Amérique [mais ce navire n'est pas arrivé à cause de la faute de Massiot etc., etc. $]^{23}$.

De plus, le caractère protestant de La Rochelle en tant que forteresse du calvinisme est connu de tous depuis le seizième siècle. C'est dans la région de La Rochelle que les Hollandais avaient fait souche au début du dix-septième siècle, à peu près à l'époque de la

18. Archives du Massachusetts à Boston, French Collection, III: 233.

19. Archives du Massachusetts à Boston, French Collection, III, «Résumé d'une lettre de Monsieur Doudyt [sic], grand vicaire de l'évêque de Québec».

20. Archives du Massachusetts à Boston, French Collection, III, De la Barre au Ministre, Québec, 4 novembre 1683.

21. Archives du Massachusetts à Boston, French Collection, III, L'évêque de Québec au Roi, Québec, 10 novembre 1683.

22. J. F. Bosher, «The Imperial Environment of French Trade with Canada, 1660-1685», English Historical Review, 108 (1993): 50-81.

23. Archives nationales, Paris, N TT 263B, folios 1056-1073, «Mémoire des choses qui se sont passées et se passent contre les ordres de Sa Majesté que les sieurs Le Court de Timonville et Coderey ont découvert dans la ville de La Rochelle...» 
fondation de la ville de Québec. Chemin faisant, bien sûr, leur influence a permis un renforcement du calvinisme dans la région. Responsables du drainage des marais d'Aunis et de Saintonge, ils s'occupaient aussi d'acheter les vins et les eaux-de-vie pour les revendre avec profit dans le nord de l'Europe. En effet, les Hollandais sont à l'origine non seulement de la commercialisation des eaux-de-vie, mais aussi de la vente de sel de Brouage dans les pays baltes. Les provinces de Saintonge et d'Aunis devinrent en grande partie calvinistes, et on identifiait les ports de pêche de ces provinces comme huguenots au dix-septième siècle. Beaucoup des navires - la majorité certaines années - engagés dans le commerce avec le Canada provenaient des petits ports de la Seudre et de la péninsule d'Arvert, dont par exemple, Marennes, La Tremblade, Mornac, Chaillevette, Royan et Talmont. Comme pour renforcer le caractère protestant de la région, des groupes de marchands hollandais et anglais protestants, dont nous connaissons parfois jusqu'aux noms, étaient encore actifs tout au long du dix-septième siècle. C'est tout près de La Rochelle, sur l'île de Ré, que les forces anglaises débarquèrent en juillet 1627 pour aider les Rochelais, expédition ratée et apparemment mal menée par le duc de Buckingham ${ }^{24}$. Bref, l'historien qui se veut conscient de l'histoire de la région de La Rochelle s'attend à y trouver des marchands protestants.

Et il n'a pas tort pour la période antérieure aux années 1670, car il y avait de ces vieilles familles calvinistes dont quelques-unes envoyaient des navires de temps à autre au Canada ou en Acadie. Les exemples ne manquent pas. En 1649, Henri Bardet prêta 20000 livres, et Samuel Pagès 10000 livres, sommes immenses à l'époque, à la Communauté de Québec ${ }^{25}$. Or, Bardet était l'un de ces huguenots très riches de La Rochelle. En 1634, il avait formé une société avec trois autres huguenots, Jacques Bibaud, Pierre Tallemant sieur Duboyneau, banquier de La Rochelle, et Abraham Dubrosse, marchand de Bordeaux, société dont le capital collectif s'élevait à 400000 livres $^{26}$. Samuel Pagès, huguenot de La Rochelle lui aussi, armait des vaisseaux pour le Canada dans les années 1650 et ses fils prêtaient des

24. Liliane Crété, La vie quotidienne à La Rochelle au temps du grand siège, 1627-1628 (Paris, Hachette, 1987), les chapitres 4 et 8; David Parker, La Rochelle and the French Monarchy: Conflict and Order in Seventeenth-Century France (London, Royal Historical Society, 1980), 15-16.

25. Marcel Delafosse, «La Rochelle et le Canada au XVII ${ }^{\mathrm{e}}$ siècle», op. cit., 476; AD Charente-Maritime, notaire Teuleron (La Rochelle), 7 juin 1649, obligation.

26. AD Charente-Maritime, notaire Chesneau (La Rochelle), 28 novembre 1634, association; Émile Magne, La joyeuse jeunesse de Tallemant des Réaux: d'après des documents inédits (Paris, 1921); Émile Magne, La fin troublée de Tallemant des Réaux (Paris, Émile-Paul Frères, 1922), chapitre II. 
fonds, en 1671, à un autre armateur rochelais de la part du gouverneur à Québec, de Courcelle ${ }^{27}$. La dynastie des Pagès était issue du mariage de Samuel en 1650 avec Anne Janssen, fille d'un grand fabricant de papier à Angoulême, huguenots eux aussi, bien sûr, mais d'origine hollandaise $^{28}$. L'historien sera inévitablement impressionné par la présence de ces gros capitalistes protestants de La Rochelle, non seulement par les Bardet, Tallemant et Pagès, mais aussi par les Manigault, D'Harriette, Théroulde, Depont, Faneuil et tant d'autres. Voilà donc l'explication du rôle exagéré attribué aux marchands huguenots dans le commerce avec la Nouvelle-France.

Cependant, pour bien comprendre la situation, il faut aussi tenir compte du progrès de la Contre-Réforme française du dix-septième siècle. À partir des années 1620, l'Église et l'État menaient une lutte de tous les instants, presque une guerre, contre les huguenots, et ceux de La Rochelle en ont souffert comme les autres. Écartés peu à peu de La Rochelle, ils ont perdu leur prépondérance dans le commerce rochelais. Trois événements capitaux au cours du siècle marquent les sommets des vagues de persécution lancées sous les règnes des Bourbons: le siège de La Rochelle en 1627-1628, l'exil en 1660-1661 de quelque 2400 huguenots de cette même ville, et la suite de la révocation de l'Édit de Nantes en $1685^{29}$. Sans atteindre l'ampleur de la révolte des Camisards dans les Cévennes au début du dix-huitième siècle, les luttes dans le sud-ouest ne peuvent que renforcer là aussi l'impression qu'ont les historiens d'une forte présence à La Rochelle de ces vieilles familles d'hérétiques «opiniastre», ainsi nommées par les autorités. Toutefois, on n'a pas tenu compte de l'effet funeste de la persécution dont ils ont été victimes et qui a beaucoup réduit leur

27. AD Charente-Maritime, notaire Teuleron (La Rochelle), 21 mai 1671 [3 E 1360], obligation à la grosse; Marcel Trudel, Histoire de la Nouvelle-France (Montréal, Fides, 1979), III: 216-218; Lionel La Berge, Rouen et le commerce du Canada de 1650 à 1670 (L'AngeGardien, Éditions Bois-Lotinville, 1972), 12, 21.

28. AD Charente-Maritime, 4 J 7, notes Garneau, s.v. «Pagès»; Gabriel Delâge, $L$ 'Angoumois au temps des marchands flamands (17 e siècle) (Paris, Bruno Sepulchre, 1990), 26-27, 30-37, 166-167.

29. Sur les effets religieux du siège, François Moisy, Le rétablissement des structures catholiques après le siège de La Rochelle, 1628-1648 (Fontenay-le-Comte, Revue du Bas-Poitou et des Provinces de l'Ouest, 1973), 5-84; sur les exilés de 1661-1662, Louis Pérouas, Le diocèse de La Rochelle de 1648 à 1724: Sociologie et Pastorale (Paris, SEVPEN, 1964), 299-307; sur les effets de la Révocation, e.g. Claude Cardoman de Rulhière, Éclaircissements historiques sur les causes de la Révocation de l'Édit de Nantes et sur l'État des Protestants en France depuis le commencement du règne de Louis XIV... (s.1., s.é., 1788), 2 tomes [l'une des meilleures études sur le sujet]; et en général, Kevin C. Robbins, «The Families and Politics of La Rochelle, 15501650», thèse de doctorat inédite, Johns Hopkins University, 1991, et Katherine L. Faust, «A Beleagured Society: Protestant Families in La Rochelle, 1628-1685", thèse de doctorat inédite, Northwestern University, 1980. 
présence et leurs activités. L'image d'une forte présence huguenote dans la région, qui était vraie dans les années 1640 et 1650, ne l'était plus pour les années 1680 et $1690^{30}$.

La base navale de Rochefort, construite dès 1666 pour relayer Brouage comme arsenal de la région, servait à la fois de lieu de contrôle des huguenots et de port militaire pour la surveillance des colonies outre-Atlantique. Pendant les années 1680 et 1690, la correspondance de l'intendant de la marine à Rochefort traite des «nouveaux convertis» et des «protestants» plus que de tout autre sujet, et de loin ${ }^{31}$. Les persécutions contre les huguenots nous sont contées complaisamment par l'un des missionnaires capucins de l'époque, Théodore de Blois, dans son Histoire de Rochefort publiée en $1733^{32}$.

Persuadé que la Religion est nécessaire pour fixer le cours du libertinage qui est rapide dans les nouvelles Colonies, [l'intendant] obtint de l'Évêque de la Rochelle, Henry de Laval, la permission de changer le Prêche que le seigneur de Rochefort avait fait bâtir pour l'exercice de la Religion Prétendüe Réformée, dont il faisait profession, dans une Chapelle, dont le soin fut donné à un Aumônier... Monsieur de Demuin fit encore paroître son zèle ardent pour la Religion dans tous les mouvemens qu'il se donna pour faire retirer les Prétendus Réformées dans le sein de l'Église. Il employa pour les ramener la douceur de l'instruction \& la sévérité des Loix. Il leur ôta tous les emplois qui étoient à sa disposition. Il obtint un Arrêt du Conseil, datté du 11 septembre 1677 qui leur défend de prendre la qualité de Fidèles, \& à leurs Pasteurs d'en prendre d'autre que celle de Ministres de la R.P.R. Il ôta toutes les distinctions de leur Temple de la Rochelle, $\&$ reduisit leurs Bancs à l'uniformité... Il chassa une infinité de Maîtres d'École, qui étoient autant de séducteurs des enfans... ${ }^{33}$

La persécution des familles dans le commerce du Canada, telle qu'elle s'est exercée contre les Faneuil et les Bonfils, a laissé des traces dans la correspondance des intendants de Rochefort ${ }^{34}$. Le catalogue presque interminable des ordonnances dirigées contre les soidisant réformés est révélateur de la conception générale qui a présidé

30. Plusieurs ouvrages en histoire canadienne ne tiennent pas compte de cette évolution: par exemple, Dale Miquelon, New France 1701-1744: "a Supplement to Europe» (Toronto, McLelland and Stewart, 1987), 126; James S. Pritchard, «Ships, Men and Commerce: a Study of Maritime Activity in New France», thèse de doctorat inédite, Université de Toronto, 1971.

31. Archives de la Marine, Rochefort, articles 1 E 35 à 1 E 43, passim.

32. Théodore de Blois, Histoire de Rochefort contenant l'établissement de cette ville, de son port et arsenal de marine, et les antiquitez de son château (Blois, chez Philbert-Joseph Masson, 1733), $281 \mathrm{p}$.

33. T. Blois, op. cit., 60-62, 70-72.

34. Archives de la Marine, Rochefort, 1 E 49 folios 23, 82, 431; 1 E 84, folios 434, 530, 533, 554 et 738 . 
à la répression ${ }^{35}$. Un recensement des familles huguenotes pendant le règne actif de Louis XIV (1661-1715) nous permet d'évaluer le nombre de familles qui sont disparues, soit par faillite, émigration à l'étranger ou par conversion forcée. Ainsi, après la faillite de Louis Pagès en $1684^{36}$, ses parents s'expatrièrent à Hambourg, en Allemagne, en Angleterre et en Hollande ${ }^{37}$. Les Faneuil s'enfuirent également mais à Rotterdam, à Boston et à la Nouvelle-York avec une partie des Manigault et des Depont, dont certains parmi ces derniers se laissèrent convertir ${ }^{38}$. Quant à Étienne D'Harriette, il est ruiné à la révocation de l'Édit de Nantes ${ }^{39}$. L'histoire des autres huguenots n'est pas très différente. Certes, certains persistaient à demeurer à La Rochelle déguisés en «nouveaux catholiques», mais l'hypocrisie de leur abjuration se manifeste au milieu du dix-huitième siècle lorsqu'ils recommencent à signer des registres protestants ${ }^{40}$. Quoi qu'il en soit, il est certain que la campagne de persécution a largement atteint ses buts à La Rochelle.

Qui alors pouvait-il bien rester dans les villes portuaires pour faire le commerce avec le Canada et l'Acadie? Les intendants des généralités de La Rochelle et de Bordeaux se plaignaient du bouleversement du commerce du sud-ouest et craignaient les conséquences

35. Eugène Guitard, Colbert et Seignelay contre la religion réformée (Paris, Auguste Picard, 1912), deuxième édition; Léon Pilatte, éd., Édits, déclarations et arrests concernans la Religion Prétendu Réformée, 1662-1751 (Paris, Fischbacher, 1885), 660 p.

36. Pierre Boissonnade et P. Charliat, Colbert et la Compagnie du Nord 1661-1689 (Paris, Rivière, 1930), 118; AD Charente-Maritime, B1612, registre du Présidial, 1684-1685.

37. Édouard Mourgue-Molines, «Les Huguenots réfugiés à Hambourg», Bulletin de l'Académie des Sciences et Lettres de Montpellier, 9 (1978): 62-73; La Haye, Gemeentearchief, fichier [immense] des huguenots réfugiés; University of Victoria, B.C., Dutch Documents, ed., Jan Kupp, tome 9, C 345, les Députés de la Compagnie du Canada à Paris à Henry Pagès, «banquier à Hambourg», 16 août 1703; Proceedings of the Huguenot Society of London, édité depuis 1885, et le «Quarto Series» de cette Société en 57 tomes.

38. AD Charente-Maritime, 2 J 103 et 103bis, Jean Paudin de Lussaudière, «État alphabétique de Protestants aunisiens et saintongeais fugitifs», fichier manuscrit; Robert Forster, Merchants, Landlords, Magistrates: the Depont Family in Eighteenth-Century France (Baltimore, The Johns Hopkins University Press, 1980).

39. AD Charente-Maritime, Notes Garnault, s.v. «D'Harriette»; notaire Drouyneau (La Rochelle), 13 janvier 1672, folio 14, mariage; AD Gironde, notaire Cazenave (Bx), 15 septembre 1681 , folio 647 , testament; 14 juin 1686 , folio $215 ; 17$ janvier 1687 , folio 360,525 concord; 5 avril 1687, folio 428, 437, testament; notaire Gariteau (LR), liasses, 5 avril 1709 [3 E 2077]; notaire Micheau (LR) 9 octobre 1698, testament; 28 décembre 1713, folio 377, testament; 3 juillet 1717, acte mortuaire; 12 juillet 1717, inventaire des papiers; 23 décembre 1717, traité; J. F. Bosher, Négociants et Navires..., op. cit., s.v. «D’Harriette».

40. AD Charente-Maritime, 2 J 94 «Fichier de mariages protestants»; et la série I des registres paroissiaux de maints villages huguenots de la région où l'on trouve des registres protestants qu'on a recommencé à tenir dans les années 1750 ou 1760 (Arvert, La Tremblade, Marennes, Mornac-sur-Seudre, Mortagne-la-vieille, Ozillac, Vaux-sur-mer); Bibliothèque municipale de La Rochelle, «Grand livre des protestants, 1731-1781». Pour Bordeaux, Archives municipales de la ville, séries GG qui témoigne du renouvellement des registres protestants dans les années 1750. 
d'une pareille oppression contre les Juifs ${ }^{41}$. Certes, depuis la conquête de la ville de La Rochelle en 1628, quelques négociants catholiques continuaient de faire le commerce transatlantique, notamment ceux du clan Tuffet-Gaigneur. Mais pendant l'expansion coloniale du règne de Louis XIV, il y avait aussi une foule de petits marchands catholiques dont Louise Dechêne et James S. Pritchard, reconnaissait l'existence, il y a vingt ans $\mathrm{s}^{42}$. Qui sont-ils et d'où viennent-ils? Le mieux connu, Antoine Pascaud (1665-1717), a été souvent cité dans les livres d'histoire, mais le milieu où il a vécu reste à peu près inconnu. Or, il trouve place parmi d'autres marchands, comme lui d'origine étonnamment modeste. Leur ascension s'avère d'autant plus intéressante que le grand commerce maritime demandait une certaine expertise, un travail assidu et des capitaux hors du commun. À ce propos, c'est sous le règne de Louis XIV, surtout dans les années 1690, que l'on a commencé à employer le mot «négociant» pour distinguer ces grands marchands actifs dans le commerce maritime des simples boutiquiers qu'on continuait à nommer «marchands». Comment donc expliquer la montée de trois simples engagés, Paul Berry, Antoine Pascaud et Pierre Plassan, de même que celle du fils d'un simple boulanger, Jean Gitton? Ou encore celle d'un petit marchand illettré, Antoine Grignon, et de deux fils de boutiquiers, Guillaume Jung de Fleurance (Gers) et Hilaire Bourgine de Poitiers, élevés dans des petites villes éloignées de la mer? Quel gouffre entre l'humble origine sociale de ces sept commerçants et celle des grandes familles, catholiques et protestantes, telles que les Gaigneur, les Bardet et autres marchands qui ont fait le commerce avec le Canada!

Pour expliquer ces carrières étonnantes, on trouve plusieurs facteurs déterminants dont quelques-uns sont communs à tous nos marchands. L'alliance conjugale avec une famille déjà active dans le commerce transatlantique était sans doute le plus décisif de ces facteurs. Le commerce canadien d'Antoine Grignon remonte au mariage de sa fille, Jeanne Grignon, avec Pierre Gaigneur, le 16 février 1654. Quelques semaines après son mariage, il effectuait le premier de ses nombreux voyages au Canada. Son intérêt pour le commerce avec la colonie ne s'est arrêté qu'à son décès en 1675; il mourut dans la maison de son gendre à La Rochelle ${ }^{43}$. Quand Jean Gitton, père, épousa Dominge Marot le 14 janvier 1657, il se liait avec le père de

41. A. M. de Boisliste, éd., Correspondance des Contrôleurs Généraux des Finances (Paris, imprimerie nationale, 1874), I: 97 et 167, Bégon au Contrôleur Général, 11 et 12 mars 1687,12 et 21 décembre 1688 .

42. Louise Dechêne, Habitants et marchands de Montréal au XVII siècle (Montréal, Plon, 1974), 214; James S. Pritchard, op. cit., 1971, passim.

43. AD Charente-Maritime, notaire Teuleron (La Rochelle) liasses, 18 décembre 1675 , inventaire après décès. 
sa nouvelle épouse, Bernard Marot, capitaine de navire connu pour ses voyages au Canada, et avec un beau-frère, Fabien Marot, capitaine de navire lui aussi ${ }^{44}$. Voici donc les Gitton intégrés dans un milieu qui connaît bien le commerce avec la Nouvelle-France. Le fils de ce Gitton, par exemple, eut comme parrain Jean Garos (ca 1616-1671), petit marchand de passage à Québec en 1644 et 1653, devenu commis et procureur général de la Communauté des Habitants de la NouvelleFrance en 1651-1652 $2^{45}$. Plus de trente ans plus tard en 1691, Jean Gitton, fils, épousa la fille d'un négociant de Bordeaux, Pierre Billatte, lui aussi dans le commerce transatlantique. Pour ce qui est du commerce canadien de Guillaume Jung il commence à peu près au moment où il se maria avec Marie Soumande, veuve de marchand et fille d'une famille très active dans le commerce avec la Nouvelle-France. Elle avait un frère capitaine de navire, Pierre Soumande de Lorme (1659-ca 1698), un autre frère, marchand à Montréal, Jean Soumande (1669-1716), et un beau-frère français, marchand à Québec, François Hazeur (1638-1708).

De judicieux mariages étaient précieux aussi pour les plus jeunes négociants. Le progrès commercial de Paul Berry était assuré le 2 décembre 1692 lorsqu'il signa un contrat de mariage à Québec avec Marie Mars, veuve de François Rivière et fille de Simon Mars, négociant bien établi dans le commerce maritime entre le Canada et la France. Ce mariage apporta à Paul Berry une tribu de beaux-frères et de cousins marchands: Raymond Duboscq à Bordeaux et à Québec, Jacques de Faye à La Rochelle, de même que Charles de Couagne, Joseph Riverin et Jean de Faye à Québec. En 1703, Jacques Leclerc (1679-1753), marchand dans le commerce avec le Canada à La Rochelle, parvenait à s'associer à ce grand clan grâce à son mariage avec Marie-Anne de Couagne. Deux autres de nos sept négociants ont épousé des femmes canadiennes qui leur ont apporté des relations utiles dans la colonie. Pierre Plassan épousa à Québec en 1695 Louise Albert, issue d'une famille dont le père était propriétaire à la côte de Lauzun et dont un des fils travaillait comme capitaine à bord de l'un des navires de Plassan, La Belonne. Ce garçon avait appris la navigation à partir de 1703 sous la protection de Plassan ${ }^{46}$. Antoine Pascaud,

44. Bibliothèque municipale de La Rochelle, paroisse Saint-Nicolas (La Rochelle) 14 janvier 1657, mariage; Joan Dawson, «Colonists or Birds of Passage? A Glimpse of the Inhabitants of La Have, 1632-1636», Nova Scotia Historical Review, 9 (1989): 54-56.

45. AD Charente-Maritime, notaire Teuleron (LR) 15 novembre 1651 et 9 janvier 1652; Marcel Trudel, Catalogue des immigrants, 1632-1662 (Montréal, Hurtubise HMH, 1983), 138, 269.

46. Archives nationales du Québec, notaire Chamballon (Québec), 30 octobre 1695, mariage; Barbel (Québec), 14 novembre 1703, convention; AD Gironde, notaire Bernard (Bx), 11 juillet 1709; notaires Marchand \& Soulard (LR), 2 juillet 1709; Sylvain Poujol et Paul de Peretti, «Un pionnier de la Nouvelle-France: Guillaume Albert (1635-1708)», L'Ancêtre, 18 (1991): 94 . 
quant à lui, épousa Marguerite Bouat (1673-1751) à Québec, femme remarquable qui diriga, pendant trois décennies, les affaires de sa famille après la mort de son mari en 1717. Seule exception à la règle, Hilaire Bourgine fit le mariage le moins lucratif en épousant la fille d'un simple vitrier rochelais, François Bellette, qui ne lui apporta que 1000 livres de $\operatorname{dot}^{47}$. Il est vrai que Bourgine avait déjà des amis canadiens, ainsi qu'on peut le constater sur son contrat de mariage qui porte les signatures de Jean-François Bourdon d'Hombourg et Pierre Soumande.

Voici une deuxième explication des succès de nos sept marchands: ils ont tous noué des relations au Canada, et six d'entre eux se sont donnés la peine de faire le voyage à la colonie, quelques-uns y sont même allés plusieurs fois. Ces nombreuses traversées de l'Atlantique témoignaient de qualités personnelles certaines, surtout de l'énergie et du courage. Il n'est pas nécessaire d'insister sur les dangers du voyage outre-Atlantique au dix-septième siècle sur ces curieux petits voiliers qu'ils appelaient «navires», dépassant rarement 80 pieds, qui prenaient six ou huit semaines pour la traversée vers le Canada et trois à cinq semaines pour le retour en France. Antoine Grignon voyagea à Québec au moins six fois dans ces conditions: en $1654,1656,1658-1659,1659-60,1661$ et $1662^{48}$. Son fils, Jean Grignon, voyageait également au Canada où sa fille épousa Louis Leber sieur de Saint Paul, natif de Montréal, fils de l'un des marchands les plus riches de la colonie ${ }^{49}$. Jean Gitton, père, quant à lui, voyagea au Canada en $1655,1656,1658-1659$, et 1667 ; son fils le fit en 1688 et à plusieurs autres occasions ${ }^{50}$. Par exemple, en juin 1674, Jean Gitton, père, conclut un marché avec Aubert de la Chesnaye par lequel il promettait d'amener leur navire L'Espérance (200 tonneaux) de La Rochelle à Québec où il devait suivre les ordres de leur partenaire, Bazire ${ }^{51}$. Selon nos informations, Guillaume Jung est le seul à n'être jamais allé au Canada, mais il y envoya un fils, un neveu

47. AD Charente-Maritime, notaire Berthellot (La Rochelle), registres, 9 juin 1687, mariage.

48. Marcel Trudel, Catalogue des immigrants, 1632-1662, 303, 330, 381, 430, 444, 477.

49. AD Charente-Maritime, notaires Rivière \& Soulard (La Rochelle), 25 avril 1689.

Pour ce mariage, Jean Grignon trouva une dot de 10000 livres dont 7000 sous forme «d'une grande maison, court, jardin, magazin, chambres, greniers et autres dépendances» dans la rue de la Verdière et de l'Escale. Parmi les témoins de ce grand mariage franco-canadien se trouvèrent les Gaigneur, les Pachot et les Gitton.

50. Bibliothèque municipale de La Rochelle, notaire Moreau (La Rochelle), 11 février 1658; AD Charente-Maritime, notaire Drouyneau (La Rochelle), 7 juin 1667; Marcel Trudel, Catalogue des immigrants..., 319, 330, 381.

51. Sa part dans cette expédition était d'un quart et la part d'Aubert, de trois-quarts (AD Charente-Maritime, notaire Teuleron (La Rochelle), 30 juin 1674, folio 117, marché). 
et, en 1692, un charpentier de barriques qui était nul autre que le futur marchand Pierre Plassan ${ }^{52}$. Plassan, selon nos documents notariés, a sûrement dû traverser l'Atlantique au moins douze fois entre 1692 et 1716. Toujours nommé «marchand demeurant ordinairement à Québec», il était néanmoins à Bordeaux, La Rochelle ou Rochefort en $1702,1705,1706,1708,1709,1711$ et $1714^{53}$. À l'instar d'Antoine Pascaud, Hilaire Bourgine a passé plusieurs années à Québec et à Montréal. Ainsi, dans le contrat de mariage qu'il signa à La Rochelle le 9 juin 1687, il est identifié comme «notaire et greffier de l'Isle de Montréal, pays de Canada y demeurant ${ }^{54}{ }^{»}$.

Le succès d'Hilaire Bourgine nous amène à relever un troisième facteur d'explication. Ce négociant, et son fils par la suite, bénéficiaient d'une relation étroite avec le clergé catholique, en France et au Canada. Sans pouvoir préciser la nature de cette relation, nous en voyons des signes un peu partout. En 1671, il acheta une rente sur le Clergé de France qui lui rapporta 116 livres 13 sols par an, et d'autres rentes semblables suivirent ${ }^{55}$. Le 8 mai 1691, il conclut une société avec Jean Sébille, négociant de Bordeaux établi à Québec où il était très actif dans les affaires religieuses ${ }^{56}$. En 1697, la belle-sœur de Bourgine, Marguerite Bellette, épousa Polycarpe Massé, procureur au siège présidial de La Rochelle, qui avait déjà servi de parrain au baptême du fils d'Hilaire Bourgine, Charles Polycarpe Bourgine ${ }^{57}$.

Le 17 décembre 1718, un autre des fils d'Hilaire Bourgine, JeanBaptiste, reçut la tonsure des mains mêmes de l'évêque de $\mathrm{La}$ Rochelle $^{58}$. En outre, à l'occasion du mariage de son fils, Charles-

52. AD Gironde, notaire Loste (Bx), 14 avril 1692, folio 59, engagement.

53. AD Gironde, notaire Fournier (Bordeaux), 8 mars 1702, folio 66; notaire Parran fils (Bx), 16 mars 1702, folio 221; notaire Guimard (Bordeaux), 27 mars 1705, folio 148; notaire Lenfumé (Bordeaux), 27 mars 1711, 26 mars 1714; AD Charente-Maritime, notaires Rivière \& Soulard (La Rochelle), 1 mai 1706, folio 174, 25 juillet 1711; AD Charente-Maritime, notaire Tayeau (Rochefort), 10 juin 1708; Guillemot (La Rochelle), 17 avril 1714, folio 238.

54. AD Charente-Maritime, notaire Berthellot (La Rochelle), registres, 9 juin 1687, mariage.

55. AD Charente-Maritime, notaire Micheau (La Rochelle), liasses, 15 décembre 1706, procuration.

56. Archives nationales du Québec, notaire Gilles Rageot (Québec), 8 mai 1691, société; AD Charente-Maritime, notaire Micheau (La Rochelle), registres, 22 janvier 1705, folio 92, cession (document particulièrement riche); Marie-Aimée Cliche, Les pratiques de dévotion en Nouvelle-France: comportements populaires et encadrement ecclésial dans le gouvernement de Québec (Québec, Université Laval, 1988), 197, 281, 287, 291.

57. Bibliothèque municipale de La Rochelle, paroisse Saint-Barthélemy (La Rochelle), 5 novembre 1696, baptême; AD Charente-Maritime, notaire Juge (LR), registres 16 février 1697, folios 60-61, mariage.

58. Ce fils devint prêtre le 21 septembre 1726. En 1752, il demeurait au Séminaire des missions étrangères, rue du Bacq à Paris, et à son décès en 1775, il était prêtre chanoine à La Rochelle (AD Charente-Maritime, notaire Solleau (La Rochelle), 5 janvier 1752, 28 mars 1752; notaire Crassous (La Rochelle), 23 janvier 1775, inventaire, 7 et 28 février 1775, inventaire). 
Polycarpe, le 10 novembre 1722 à Poitiers, c'est son frère chanoine de La Rochelle, René-Hilaire Bourgine, qui célébra ce mariage ${ }^{59}$. À la même époque, Charles-Polycarpe commença à remplacer son père dans le commerce avec le Canada. Enfin, en 1729, un visiteur au Canada parlait dans une lettre de «Monsieur Bourgine que vous scavez, qui a soin des missionnaires du Canada ${ }^{60} .{ }^{\circ}$ D'ailleurs, la France accordait des sommes considérables à l'Église naissante du Canada et de l'Acadie sous le règne de Louis XIV ${ }^{61}$.

Cependant, Bourgine n'était ni le seul ni le premier des sept négociants à avoir des relations étroites avec le clergé. Le 20 février 1657, Antoine Grignon se proposait d'envoyer à Québec «une balle enquessée couverte de paille et taille par dessus et cordée en plusieurs endroits sur le dessus et à un bout y a escript en grosses lettres $A u x$ révérendes mères Ursulines à Québec et y a escript trois cent cinquante et marquée de la marque cy en marge dont partant de lad. balle led. Grignon se contente...» Il avait signé cet acte en présence de Pierre Gaigneur et Marie de l'Incarnation avait commandé ces marchandises $^{62}$. Quant à Guillaume Jung, il gagna par son mariage avec Marie Soumande, en 1684, à la fois un beau-frère déjà prêtre qui deviendra bientôt chanoine de la cathédrale de Québec et une bellesœur élève des Ursulines de Québec, qui fut plus tard la première supérieure de l'Hôpital général de Québec. Deux de ses filles devinrent Ursulines, et il avait une foule d'autres parents dans les ordres en France et au Canada ${ }^{63}$. Pour ce qui est de Jean Gitton, père, une de ses filles est devenue religieuse hospitalière le 13 février 1678 , ce qui lui a coûté 3000 livres; il avait déjà offert des sommes d'argent à l'Église, telle la donation de 220 livres 13 sols à la paroisse SaintNicolas $^{64}$. Paul Berry, de son côté, avait un frère, Jean-Pierre Berry, «prestre docteur en théologie, chanoine de l'église cathédrale de la Rochelle, curé de Longesue et prieur du Prieuré Saint-Romel de Châtellaillon ${ }^{65} . »$ Enfin, il semble bien qu'Antoine Pascaud et Pierre

59. Bibliothèque municipale de Poitiers, paroisse Saint-Savin (Poitiers), registre 231, folio 55,10 novembre 1722 , mariage.

60. Archives Nationales du Canada, Archives du Séminaire des Missions Étrangères, Bobine C - 14015, Saint-Sulpice (Paris), «Relation du voyage de Canada de l'année 1729 par Pierre-Thomas Ruffin de la Morandière», folio 188.

61. Claude Lessard, «L'aide financière de l'Église de France à l'Église naissante du Canada", Mélanges d'histoire du Canada français offerts au professeur Marcel Trudel (Ottawa, Presses de l'Université d'Ottawa, 1978), 162-181; Guy Frégault, Le XVIII siècle canadien: études (Montréal, Hurtubise HMH, 1968), chapitre III, «L’Église et la société canadienne».

62. AD Charente-Maritime, notaire Teuleron (La Rochelle), liasses, 18 décembre 1675 (3 E 1364), inventaire; notaire Cherbonnier (La Rochelle), liasses, 20 février 1657.

63. Voir notre article, «Une famille de Fleurance...», op. cit., 166-167.

64. AD Charente-Maritime, notaire Drouyneau (La Rochelle), liasses, 25 avril 1677, donation; notaire Teuleron (LR), liasses, 5 février 1678, quittance.

65. AD Charente-Maritime, notaire Grenot (La Rochelle), 20 janvier 1710, procuration, 22 février 1710 , folio 148 , transaction. 
Plassan aient été des exceptions à cet égard, car nous n'avons trouvé dans leur famille aucune trace de frères ou de fils parmi le clergé.

Quoi qu'il en soit, Plassan a encaissé une bonne somme d'une société qu'il conclut le 13 novembre 1703 à Québec avec un certain Jean Petit, agent des trésoriers généraux de la Marine ${ }^{66}$. Voici donc un quatrième facteur de réussite pour ces négociants: de bonnes relations, louches parfois, avec des gens en autorité. En fait, Plassan et Petit touchèrent un capital de 15000 livres, dont Petit versa la moitié à son associé «en lettres de change tirées sous le nom dudit Sieur Plassan». Petit abusait ainsi de sa place, à la façon classique de l'ancien régime, en utilisant à des fins personnelles le crédit qu'il pouvait obtenir comme agent des trésoriers généraux. Le gouvernement, sans doute, aurait fermé les yeux sur une telle société pendant la guerre de la succession d'Espagne. Mais à cette époque, Petit se méfiait et seul Plassan devait signer tout document d'affaires de la part de la société:

Et autant que ledit Sieur Petit ne veut pas que son nom soit employé dans les factures et connaissements que ledit Sieur Plassan tirera desdites marchandises, elles ne laisseront cependant d'être pour le compte et risque de lad. société aussi bien que le profit d'icelles, encore que le tout ne soit fait que sous le nom dudit Plassan seul ${ }^{67}$.

Les actes notariés exigeant la signature de témoins, Henry Hiché, commis au magasin du roi à Québec, les signa. Selon l'acte de société, Plassan devait employer les 15000 livres ainsi obtenues en France pour acheter «de(s) marchandises qu'il estimera les plus propres et convenables pour ce paÿs où il les envoiera par un des navires qu'il verra être le plus sûr de ceux qui partiront de France pour revenir en ce dit paÿs l'an prochain, 1704, lesquelles marchandises il pourra risquer ou faire assurer selon qu'il jugera plus à propos». Au mois d'octobre 1705, ce capital avait atteint la somme de 44378 livres et à la dissolution de cette société, trois ans plus tard, les associés comptaient parmi leurs avoirs plusieurs parts de navires. Ce capital comprenait un quart du Chamilly valant 13000 livres, un fonds de 3500 livres à Plaisance pour les achats de morues, des marchandises pour 4000 livres chargées dans La Notre Dame des Victoires, navire qu'ils possédaient d'ailleurs en partie, environ 5000 livres en pelleteries que Plassan comptait alors apporter avec lui en France et plusieurs créances sur des marchands au Canada et en

66. Archives nationales du Québec, notaire François Genaple (Québec), 13 novembre 1703, acte de société.

67. Ibid., 16 novembre 1704 , renouvellement de la société. 
France ${ }^{68}$. Il convient d'ajouter que Petit avait un frère qui était contrôleur des rentes à l'hôtel de ville de Paris, et un beau-frère, Simon Dupuy, enseigne d'une compagnie des troupes du détachement de la Marine au Canada, qui semblent être impliqués, eux aussi, dans les affaires transatlantiques de la société69.

Combien d'autres de nos négociants profitaient d'arrangements pareils? Il est malheureusement de la nature d'ententes comme celle de Plassan et Petit d'être faites discrètement, mais nous n'en avons pas trouvé d'autres exemples dans les transactions de nos négociants. Du moins pas encore quoique certains arrangements soulèvent des points d'interrogation. Que faisait Jean Gitton, père, entre le 3 septembre et le 22 décembre 1685, lorsqu'il visitait Paris dans le but de conférer avec Colbert de Seignelay «pour le bien public et utillité du négoce ${ }^{70} \gg$ ? Les juges et consuls de La Rochelle l'avaient choisi, le 23 août 1684, pour les représenter à cette réunion suivant les ordres du roi. Plus tard, en 1712, Jean Gitton, fils, faisait des affaires avec l'intendant du Canada, Bégon, et son associé Butler de La Rochelle, mais nous ne connaissons pas le détail de leurs transactions ${ }^{71}$. Nous en sommes donc réduit à ne pouvoir identifier que Pierre Plassan parmi nos négociants ayant eu des relations louches avec les autorités.

Les guerres de Louis XIV, cinquième et dernier facteur, ont certainement créé des circonstances favorables au progrès de six des négociants retenus. Certes, Antoine Grignon décéda en 1675, avant la guerre quasi mondiale de la Ligue d'Augsbourg (1689-1697), mais il avait peut-être tiré certains avantages des préparatifs impériaux de Louis XIV en vue de ses grandes guerres avec la Hollande, l'Angleterre et leurs colonies en Amérique ${ }^{72}$. La génération de Berry, Pascaud et Plassan a joui d'un commerce colonial presque toujours florissant pendant les deux guerres maritimes, de 1689 à 1713. Ce sont en partie des guerres commerciales où le concours de la Hollande et de l'Angleterre jouait un rôle important. Pour ces deux puissances alliées, le conflit originait en partie des tarifs de 1667 et 1671 imposés sur leurs marchandises entrant en France. Par surcroît, ces deux pays résistaient aux empiètements de la France en Europe et dans le monde en géné-

68. Ibid., 30 avril 1707, prorogation de la société; 20 octobre 1708, dissolution de la société; 12 novembre 1708, «acte des effets qui sont en nature de la société qui étoit entre les Sieurs Petit et Plassan».

69. Ibid., 13 novembre 1703, obligation du sieur Dupuy.

70. AD Charente-Maritime, notaire Berthellot (La Rochelle), liasses, 3 juillet 1685 [3 E 2183].

71. AD Charente-Maritime, notaires Rivière \& Soulard (La Rochelle), 8 avril 1715, folio 14 , transport.

72. J. F. Bosher, «The Imperial Environment...», op. cit., 50-81. 
ral; Guillaume III avait compris que Louis XIV comptait s'imposer partout, à commencer par la Hollande qu'il avait envahie en 1672.

Cependant, aux motivations commerciales et diplomatiques, il convient d'ajouter les causes religieuses à l'origine de ces guerres. Beaucoup moins connues et mal comprises, elles provenaient de la résistance protestante aux agressions issues de la Contre-Réforme française, agressions menées aussi bien à l'extérieur qu'à l'intérieur du territoire français depuis les années 1620, et même auparavant. Car les conflits religieux en France, tels qu'illustrés par la révocation de l'Édit de Nantes en 1685 et plus tard par les guerres dans les Cévennes, avaient leur contre-partie dans les relations avec les royaumes étrangers, puisque Louis XIV voulait s'imposer comme chef de l'Europe catholique. N'oublions pas que les visées impériales de la France servaient les intérêts du clergé car, en cas de victoire, en Amérique comme en Europe, ce dernier était appelé à s'emparer des pays conquis tout autant que les armées. À cette époque, missionnaires et soldats se battaient presque ensemble pour la France. Les instructions du roi à Frontenac, datées du 7 juin 1689 à propos de l'invasion de la colonie de New York, le prouvent clairement:

Si parmy les habitants de la Nouvelle-York, soit anglois ou hollandois, il se trouve des catholiques, de la fidelité desquels il [Frontenac] croye se pouvoir asseurer, il pourra les laisser dans leurs habitations après leur avoir fait prester serment de fidélité à Sa Majesté, bien entendu qu'il n'y en ait pas un trop grand nombre... A l'égard de tous les autres estrangers, hommes, femmes et enfans, Sa Majesté trouve à propos qu'ils soient mis hors de la colonie et envoyez à la Nouvelle-Angleterre, à la Pensilvanie, ou en d'autres endroits qu'il jugera à propos par mer ou par terre... Il envoyera en France les François fugitifs qu'il y pourra trouver et particulièrement ceux de la Religion prétendue réformée ${ }^{73} . »$

Naturellement en Hollande, en Angleterre et dans les colonies anglaises, on redoutait de plus en plus les visées impérialistes et catholiques de Louis $\mathrm{XIV}^{74}$. Ces craintes existaient bien avant le règne de ce roi, mais surtout depuis que la France avait gagné ses guerres contre les Habsbourg en 1659. En effet depuis lors, Louis XIV se rangeait de plus en plus souvent du côté des dévots français. Les

73. Rapport de l'archiviste de la province de Québec, 8 (1927-28): 12-16, Mémoire [de Louis XIV] pour servir d'instruction à Monsieur le comte de Frontenac sur l'entreprise de la Nouvelle-York, 7 juin 1689; voir aussi Marcel Trudel, «Louis XIV et son projet de déportation, 1689», Revue d'histoire de l'Amérique française, 4,1 (juin 1950): 157-171.

74. J. F. Bosher, «The Franco-Catholic Danger, 1660-1715», History: Journal of the Historical Association, 79 (février 1994): 5-30. 
huguenots réfugiés en Hollande, en Angleterre et dans ses colonies attisaient cette peur dans la population de ces pays. Et qui plus est, des huguenots combattaient dans les armées protestantes contre la France. On ignore généralement l'importance de cet apport qui a presque transformé ces guerres en guerre civile entre les huguenots et les autorités monarchique et religieuse. Dès 1690 , cinq régiments huguenots d'origine française se battaient contre les armées françaises en Irlande et sur le continent. De plus, leurs capitaux affluaient vers Amsterdam et Londres pour appuyer l'effort de guerre de ces pays. Plusieurs des riches marchands et banquiers huguenots participèrent à la création de la Banque d'Angleterre en 1694 et d'autres institutions financières qui devaient assurer la supériorité de l'Angleterre durant tout le dixhuitième siècle ${ }^{75}$. Dans les colonies aussi, à Boston et à New York, des réfugiés huguenots se rangeaient du côté des puissances protestantes. Sans exagérer l'importance de la motivation religieuse dans ces conflits, il convient de la signaler, car elle est souvent ignorée dans l'histoire du commerce et des guerres à cette époque. Mais il y a une raison encore plus précise d'évoquer l'explication religieuse.

Le gouvernement de Louis XIV, en pleine guerre contre les huguenots en France depuis le mois d'octobre 1685, n'avait aucune confiance dans les négociants protestants. C'est qu'il soupçonnait et avec raison - qu'ils avaient des liens très étroits avec des parents ou des amis réfugiés dans les pays ennemis ${ }^{76}$. Après tout, comment se fier à des familles telles que les Faneuil, les Bonfils, les D'Harriette et tant d'autres, alors qu'on leur avait formellement interdit de sortir de la France? Comment confier les munitions et les forces militaires destinées au Canada à des négociants dont on voulait forcer les familles à abjurer sous peine de les mettre en prison ou de les envoyer aux galères? Certes, une partie de la communauté huguenote s'était convertie, mais une autre avait pris le chemin de l'exil pour renforcer la cause protestante. Voilà les circonstances politico-religieuses dans lesquelles le commerce avec le Canada de six de nos sept négociants catholiques prirent de l'expansion. Sans ces guerres, il est fort possible que leurs échanges aient périclité, car le commerce des fourrures était en crise entre 1690 et $1715^{77}$.

75. François Crouzet, «Les Huguenots et la révolution financière en Angleterre», Bulletin de la Société d'Histoire Moderne, $17^{\mathrm{e}}$ série, 89e année, 3 (1990): 19-25; P. G. M. Dickson, The Financial Revolution in England: a Study in the Development of Public Credit, 1688-1756 (London, MacMillan, 1967); Alice Carter, «The Huguenot Contribution to the Early Years of the Funded Debt», Proceedings of the Huguenot Society of London, 19 (1953-59): 21-41.

76. Archives de la Marine, Rochefort, 1 E 49, folio 23 (1703), 1 E 77, folio 12 (1712); Francine Miot, La Révocation de l'Édit de Nantes et les Protestants jusqu'à l'Édit de tolérance (1787) en Aunis et en Saintonge, thèse de l'École des Chartes, Paris, 1968 [AD CharenteMaritime, 4 J 2995], 133.

77. Guy Frégault, op. cit., chapitre V, «La Compagnie de la Colonie». 
Prenons, par exemple, le cas des Jean Gitton, père et fils, qui investirent largement dans la Compagnie du Nord ou de la Baye d'Hudson dans les années 1680 et qui ont soutenu l'effort militaire du roi de France. Ainsi, le fils (son père étant décédé) secondait Pierre Le Moyne d'Iberville dans ses efforts héroïques pour écarter les Anglais de la baie d'Hudson et de Terre-Neuve ${ }^{78}$. Le père avait confié ses investissements à son fils, y inclus plusieurs créances sur Cavelier de la Salle et autres particuliers, en plus d'une maison à Montréal et de marchandises canadiennes ${ }^{79}$. En janvier 1690 , Gitton, fils, acheta pour la Compagnie le navire de 100 tonneaux La Famille pour 8100 livres $^{80}$. Le 5 mai 1696, il s'engageait avec Le Moyne d'Iberville à fournir 34020 «rassions pour la subsistance des équipages des vaisseaux du Roy, L'Envieux et Le Profond», qu'il destinait à Plaisance. Il devait envoyer ces «rassions» d'une valeur de 13608 livres sur Le Postillon et Le Hardy ${ }^{81}$. Moins de quatre ans plus tard, le 18 octobre 1699, Joseph Le Moyne de Sérigny, lieutenant de vaisseau du roi, signa à La Rochelle au nom de son frère, Le Moyne d'Iberville, un marché avec Jean Gitton, fils, et un associé lyonnais, Martin Desgarinières ${ }^{82}$. Ces deux négociants devaient verser aux frères Le Moyne 32000 livres pour profiter de la moitié de la traite des fourrures du fort Bourbon (Baie d'Hudson) effectuée depuis 1698. Cette somme était due avant la fin de l'année 1700, et les frères Le Moyne devaient envoyer toutes les fourrures à La Rochelle au profit des deux négociants. Puis, à partir de 1701, les négociants devaient bénéficier de la moitié de la traite au prix de 12000 livres par an, prix qui se réduisait à 8000 livres s'ils devaient fournir le transport entre la Baye et La Rochelle. Quoi qu'il en soit, les deux négociants devaient fournir deux navires par an. Pendant la guerre suivante, de 1702 à 1713, Gitton, fils, et deux de ses associés armèrent au moins cinq vaisseaux du roi, L'Oriflame, L'Atalante, La Diane, La Valleur (?) et La Vénus ${ }^{83}$. Ces exemples montrent à l'évidence que Jean Gitton, fils, se prêtait aux visées expansionnistes du roi et de la Compagnie de la Baye du Nord.

78. AD Charente-Maritime, notaire Berthellot (La Rochelle), liasses, 22 août 1690, inventaire après décès, citant Rageot (Québec), 10 novembre 1687. Il paraît qu'en 1690, les Gitton évaluaient leur investissement à 17726 livres 13 sols.

79. AD Charente-Maritime, notaire Berthellot (La Rochelle), registres, 17 avril 1688, reconnaissance et ratification.

80. AD Charente-Maritime, notaire Martin (La Rochelle), 12 janvier 1690, folio 40, vente.

81. AD Charente-Maritime, notaires Rivière \& Soulard (La Rochelle), 5 mai 1696, folio 172, marché.

82. AD Charente-Maritime, notaire Gariteau (La Rochelle), registres, 18 octobre 1699, folio 283 , marché (2 pages).

83. AD Charente-Maritime, notaire Micheau (LR), liasses, 21 août 1720, cession [3 E $1537]$. 
Quant aux autres négociants, il est difficile de connaître les cargaisons des navires, mais il semble que les commerces transatlantiques de Paul Berry, Hilaire Bourgine, Antoine Pascaud et Pierre Plassan servaient là aussi les ambitions de la monarchie et de l'Église. Toutefois, il est certain que Pierre Plassan signa des contrats à Rochefort le 19 mai 1708 avec l'intendant de la Marine, Michel Bégon $^{84}$.

Si les dépenses de la monarchie et de l'Église enrichirent nos sept négociants pendant les guerres, certains d'entre eux durent certainement payer le prix de leur dépendance. Il y a un livre à écrire à propos de l'exploitation mutuelle de la monarchie française et de ses négociants ${ }^{85}$. Cette exploitation s'est faite à peu près sous la même forme pendant chacune des guerres maritimes entre 1689 et 1713 . Au début de la guerre, la monarchie engageait des négociants pour transporter des marchandises et des armes aux colonies et, naturellement, le prestige, l'influence et les promesses ne manquaient pas d'en tenter plusieurs. Les négociants faisaient alors d'emblée crédit à la monarchie, mais ce crédit s'épuisait petit à petit jusqu'au moment où il n'y avait ni fonds ni crédit disponibles, notamment en 1705, 1748 et 1759. Les raisons de cet affaissement du crédit de l'État sont assez complexes, mais il est certain que la défaite y est pour quelque chose. Par contre, l'effet de la victoire est plus difficile à connaître, étant donné que les victoires durables sur mer étaient plutôt rares. Quoi qu'il en soit, à la fin de chaque guerre et souvent bien longtemps après, on trouve les négociants tentant de se faire rembourser par l'État. En général, sans succès, de sorte que beaucoup d'entre eux ont fini par faire faillite. Nous avons raconté ailleurs le sort de la Veuve La Maignière qui, après avoir armé plusieurs expéditions pour le Canada pendant la guerre de la succession d'Espagne, n'a pu réussir à se faire rembourser par l'État ${ }^{86}$. À la lumière de ces faits, comment alors nos sept négociants ont-ils pu traverser les deux guerres qu'ils ont vécues?

Pierre Plassan fit faillite en 1716 et mourut quelques mois plus $\operatorname{tard}^{87}$. Lorsque Paul Berry décéda le 22 février 1709, sa veuve racheta pour 3700 livres la moitié de l'héritage léguée aux deux frères de feu

84. AD Charente-Maritime, notaire Tayeau (Rochefort), 10 juin 1708.

85. Voir notre article, «Partenaires de la Marine Royale dans le ravitaillement du Canada, 1701-1713», Rochefort et la mer, troisième partie, «Présence maritime française aux XVI et XVIII ${ }^{e}$ siècles», Colloque du 26-31 mai 1986, Université francophone d'été, Rochefort, 1986, 43-51.

86. Ibid.

87. Archives nationales du Québec, notaire La Cetière (Québec), 20 mars 1716, inventaire de Pierre Plassan et Louise Albert (15 pages). 
son mari, ce qui laisse penser que Berry était loin d'être riche ${ }^{88}$. En tout cas, il n'avait aucun enfant pour prendre le relais de son commerce transatlantique. Les familles Grignon et Gitton ont disparu également de ce commerce. Jean Grignon, fils d'Antoine, est mort en 1703, c'est-à-dire bien avant d'avoir pu jouer un rôle dans la guerre de 1702-1713. N'ayant pas couru ces risques, il était suffisamment riche en 1702 pour offrir une dot de 10000 livres à sa fille, Marianne Grignon, lors de son mariage avec «Charles Fleury, sieur de Chambeau, demeurant à La Rochelle, natif de l'Isle d'Orléans au Canada ${ }^{89}$ ». Jean Gitton, fils (1662-1717), souffrait des créances non honorées par l'État, comme Plassan et beaucoup d'autres. Trois ans après son décès, sa veuve a vendu ses créances sur l'État moyennant 15000 livres en billets de la banque de John Law - très peu de temps avant la chute de cette banque ${ }^{90}$. Malgré cette perte, elle a pu néanmoins survivre sans trop de mal $^{91}$.

Quant aux familles Bourgine, Jung et Pascaud, elles ont repris le commerce du Canada après le Traité d'Utrecht (1713). Par contre, les chefs des deux premières familles ont fait faillite: Charles-Polycarpe Bourgine pendant la guerre suivante, en 1745, et Jean Jung peu après, en $1753^{92}$. De nos sept négociants, Antoine Pascaud fut le seul à pouvoir fonder une famille idéale selon les normes de l'époque. De retour en France vers 1709, il est décédé en 1717 laissant à sa veuve canadienne suffisamment de capitaux pour continuer le commerce avec le Canada jusqu'à ce que ses deux fils atteignent un âge raisonnable pour pouvoir prendre le relais. Ces derniers n'ont pas fait faillite, comme tant d'autres, même après la perte de la colonie en 17591763. Leurs enfants et petits-enfants se lièrent par mariage avec quelques familles distinguées, voire des familles nobles, mariages dont leur grand-père aurait pu difficilement rêver pour eux alors qu'il travaillait à Québec comme engagé dans les années $1680^{93}$. Les Pascaud semblent donc être la seule de nos sept familles à avoir réussi à perdurer dans le commerce au dix-huitième siècle.

88. AD Charente-Maritime, notaire Grenot (LR), registres, 22 février 1710, folio 148, transaction, citant Chambellon (Québec), 2 février 1692 et 6 octobre 1694, etc.

89. AD Charente-Maritime, notaires Rivière \& Soulard (La Rochelle), registres, 3 août 1702, folio 187, mariage. Jean Grignon (1670-1702), fils de Jean Grignon et petit-fils d'Antoine, était décédé en 1702 .

90. AD Charente-Maritime, notaire Micheau (La Rochelle), liasses, 21 août 1720, cession [3 E 1537].

91. AD Charente-Maritime, notaire Desbarres (La Rochelle), liasses, $1^{\text {er }}$ avril 1717 , inventaire après le décès de Jean Gitton.

92. J. F. Bosher, Négociants et navires du commerce..., op. cit., 49 et 85.

93. J. F. Bosher, The Canada Merchants, 1713-1763 (Oxford, Oxford University Press, 1987), 57-60. 
$\mathrm{Au}$ terme de cette analyse, il importe de récapituler les raisons apportées au succès commercial, sous le règne de Louis XIV (et un peu avant quant à Antoine Grignon), des sept négociants étudiés. Leur réussite s'avère d'autant plus remarquable quand on la compare aux fins moins heureuses de la plupart de leurs descendants. De plus, l'importance qu'ils ont acquise dans ce commerce (quelque $27,7 \%$ du total des voyages) malgré leur origine modeste appelle une explication. Certes, on pourrait alléguer que les générations du règne de Louis XIV firent preuve de qualités dont leurs descendants auraient été dépourvus. C'est peu vraisemblable, et il serait impossible pour l'historien de prouver une telle différence entre deux générations. Nous croyons plutôt qu'il faut chercher des explications dans le contexte militaire et politico-religieux de la période allant environ de 1660 à 1715 . Le commerce français avec les colonies de la NouvelleFrance était alors en pleine expansion grâce à la politique impériale du Roi Soleil. La persécution de plus en plus vive des huguenots décourageait les marchands protestants de La Rochelle et de Bordeaux, dont les activités à Québec avaient d'ailleurs été restreintes depuis la fondation de la Compagnie des Cent-Associés en 1627. Par contre, l'importance des missions catholiques outre-Atlantique, dont l'Acadie et le Canada furent à notre sens l'expression, créait un environnement favorable à l'esprit d'entreprise chez des catholiques qui, tout en détenant les qualités nécessaires pour le commerce, étaient prêts à servir la monarchie et le clergé. Dès le début des guerres impériales contre les puissances protestantes des Pays-bas et des iles britanniques, les marchands protestants devenaient suspects à cause de leurs multiples relations avec leurs congénères réfugiés à Amsterdam, Boston, Londres, New York et Rotterdam. Au même moment, la monarchie dépensait des sommes considérables pour l'appui et la défense des colonies. Sous ces conditions, il suffisait pour un Berry, un Grignon, un Gitton, un Jung, un Plassan de s'allier à une famille déjà établie dans les échanges transatlantiques pour profiter de l'expansion commerciale. En plus, certains d'entre eux, surtout Bourgine, Grignon et Jung, avaient, d'une façon ou d'une autre, des relations privilégiées avec le clergé. Malheureusement pour ces nouveaux marchands, les moyens de la monarchie s'épuisaient après chaque guerre, et les affaires des marchands en subirent les contrecoups. De rudes épreuves les attendaient à la fin de chacune de ces guerres. Les Bourgine, les Jung et les Pascaud les surmontèrent, mais il n'y a eu véritablement que la famille de ce dernier à être active dans le commerce de la Nouvelle-France jusqu'à la défaite de 1759-1760. Ce qui ne doit pas faire oublier cependant qu'au moins sept marchands catholiques, d'origine fort modeste, ont réussi une carrière exceptionnelle sous le règne de Louis XIV. 


\section{Annexe}

Nombre de navires effectuant du commerce avec la Nouvelle-France

$\begin{array}{lccccc}\text { Année } & \begin{array}{c}\text { Tous les } \\ \text { Navires }\end{array} & \begin{array}{c}\text { navires } \\ \text { des sept }\end{array} & \text { Année } & \begin{array}{c}\text { Tous les } \\ \text { navires }\end{array} & \begin{array}{c}\text { Navires } \\ \text { des sept }\end{array} \\ 1660 & 3 & 1 & 1688 & 18 & 6 \\ 1661 & 6 & 0 & 1689 & 15 & 12 \\ 1662 & 11 & 0 & 1690 & 15 & 10 \\ 1663 & 7 & 1 & 1691 & 18 & 2 \\ 1664 & 6 & 0 & 1692 & 13 & 3 \\ 1665 & 13 & 0 & 1693 & 12 & 3 \\ 1666 & 11 & 0 & 1694 & 5 & 2 \\ 1667 & 11 & 0 & 1695 & 12 & 4 \\ 1668 & 4 & 1 & 1696 & 16 & 3 \\ 1669 & 8 & 0 & 1697 & 8 & 2 \\ 1670 & 7 & 1 & 1698 & 13 & 4 \\ 1671 & 9 & 2 & 1699 & 10 & 2 \\ 1672 & 8 & 2 & 1700 & 14 & 2 \\ 1673 & 10 & 1 & 1701 & 5 & 2 \\ 1674 & 4 & 1 & 1702 & 9 & 2 \\ 1675 & 7 & 0 & 1703 & 6 & 1 \\ 1676 & 5 & 0 & 1704 & 14 & 0 \\ 1677 & 4 & 2 & 1705 & 10 & 2 \\ 1678 & 8 & 3 & 1706 & 2 & 1 \\ 1679 & 11 & 3 & 1707 & 4 & 2 \\ 1680 & 7 & 1 & 1708 & 11 & 3 \\ 1681 & 4 & 2 & 1709 & 13 & 3 \\ 1682 & 11 & 1 & 1710 & 14 & 2 \\ 1683 & 9 & 3 & 1711 & 8 & 2 \\ 1684 & 16 & 5 & 1712 & 5 & 0 \\ 1685 & 14 & 5 & 1713 & 8 & 0 \\ 1686 & 13 & 6 & 1714 & 15 & 0 \\ 1687 & 13 & 6 & 1715 & 10 & 1 \\ & & & \text { Total: } & 443 & 123\end{array}$

